\title{
Video Article \\ Spin Saturation Transfer Difference NMR (SSTD NMR): A New Tool to Obtain Kinetic Parameters of Chemical Exchange Processes
}

\author{
María Teresa Quirós ${ }^{1}$, Colin Macdonald ${ }^{1,2}$, Jesús Angulo ${ }^{2}$, María Paz Muñoz ${ }^{1}$ \\ ${ }^{1}$ School of Chemistry, University of East Anglia \\ ${ }^{2}$ School of Pharmacy, University of East Anglia
}

Correspondence to: María Teresa Quirós at mteresaquiroslopez@gmail.com, Colin Macdonald at c.macdonald@uea.ac.uk, Jesús Angulo at J.Angulo@uea.ac.uk, María Paz Muñoz at M.Munoz-Herranz@uea.ac.uk

URL: https://www.jove.com/video/54499

DOI: doi:10.3791/54499

Keywords: Chemistry, Issue 117, NMR, spin saturation transfer, saturation transfer difference, kinetics, chemical exchange, NOE, thermodynamic parameters, SSTD NMR

Date Published: $11 / 12 / 2016$

Citation: Quirós, M.T., Macdonald, C., Angulo, J., Muñoz, M.P. Spin Saturation Transfer Difference NMR (SSTD NMR): A New Tool to Obtain Kinetic Parameters of Chemical Exchange Processes. J. Vis. Exp. (117), e54499, doi:10.3791/54499 (2016).

\section{Abstract}

This detailed protocol describes the new Spin Saturation Transfer Difference Nuclear Magnetic Resonance protocol (SSTD NMR), recently developed in our group to study processes of mutual-site chemical exchange that are difficult to analyze by traditional methods. As the name suggests, this method combines the Spin Saturation Transfer method used for small molecules, with the Saturation Transfer Difference (STD) NMR method employed for the study of protein-ligand interactions, by measuring transient spin saturation transfer along increasing saturation times (build-up curves) in small organic and organometallic molecules undergoing chemical exchange.

Advantages of this method over existing ones are: there is no need to reach coalescence of the exchanging signals; the method can be applied as long as one signal of the exchanging sites is isolated; there is no need to measure $T_{1}$ or reach steady state saturation; rate constant values are measured directly, and $T_{1}$ values are obtained in the same experiment, using only one set of experiments.

To test the method, we have studied the dynamics of the hindered rotation of $N, N$-dimethylamides, for which much data is available for comparison. The thermodynamic parameters obtained using SSTD are very similar to the reported ones (spin-saturation transfer techniques and line-shape analysis). The method can be applied to more challenging substrates that cannot be studied by previous methods.

We envisage that the simple experimental set up and the wide applicability of the method to a great variety of substrates will make this a common technique amongst organic and organometallic chemists without extensive expertise in NMR.

\section{Video Link}

The video component of this article can be found at https://www.jove.com/video/54499/

\section{Introduction}

Chemical exchange commonly refers to any intermolecular or intramolecular process in which a nucleus moves from one environment to another in which its NMR parameters (chemical shift, scalar coupling, dipolar coupling, relaxation rate) differ. There are numerous examples of chemical exchange in organic and organometallic molecules (e.g., rotational barriers in biaryls, ring flipping barriers and conformational equilibrium, nitrogen inversion, ligand binding, degenerate ligand exchange and tautomerization). ${ }^{1-3}$ The chemical exchange rate is related to the thermodynamics of the barrier of the exchange process, and therefore its study is of crucial importance to understand molecular dynamics of these systems.

The classic sign of dynamic exchange in NMR is a dramatic change in the line-shape of the NMR signals as the temperature changes. At low temperatures, the process is slow and two distinct chemical shifts are observed. At high temperatures, the two signals merge into one signal, which is known as "coalescence". At intermediate temperatures, the signals become very broad. This sensitivity of the NMR spectrum to chemical exchange makes NMR a very powerful method to study the dynamics of molecules in solution. Two methods have been mainly employed in the study of dynamic processes in solution: line-shape analysis, ${ }^{4-7}$ and spin saturation transfer experiments. ${ }^{8-9}$ Besides, it is also worth mentioning the inversion transfer method ${ }^{10}$ and the CIFIT program ${ }^{11}$ for the direct extraction of rate constants, that are a relatively efficient approach for exchange measurements in simple systems. Although these methods give very good results in most cases, they, however, have a number of drawbacks. The main disadvantage of the line-shape analysis is the high temperatures needed to reach coalescence in some samples. ${ }^{12}$ The main issues to consider when carrying out spin saturation transfer experiments are: the very long saturation times required to reach the steady state saturation transfer between the exchanging sites, and the need to determine the longitudinal relaxation time constant, $T_{1}$, which can be difficult if there is overlap of different signals in the region of study. ${ }^{13}$ 
As part of our investigations in organometallic mechanisms, ${ }^{14-16}$ our group is studying the fluxional behavior of platinum-allene complexes in solution. This is a complex task that involves at least three different processes, one of them being the $\pi$-face exchange or rotation of the metal around one of the allene axis. We encountered that normal VT experiments and line-shape analysis techniques that have been employed before in similar systems, ${ }^{17-19}$ were not suitable in our study, due to a very slow rotation in our platinum-allene complex that made the coalescence temperature of the signals of interest higher than the temperature of decomposition of the complex.

In order to overcome this limitation, we developed and recently reported a new NMR protocol (SSTD NMR) to study processes of mutual-site chemical exchange. ${ }^{20}$ As the name suggests this method combines the Spin Saturation Transfer method used for small molecules, with the Saturation Transfer Difference NMR method employed for the study of protein-ligand interactions, ${ }^{21-24}$ by measuring transient spin saturation transfer along increasing saturation times (build-up curves) in small molecules undergoing chemical exchange.

With this new method (SSTD NMR) we have shown that we can obtain the kinetic parameters of intramolecular chemical exchange in small organic and organometallic molecules with some additional advantages over traditional approaches: coalescence of the signals is not needed, so a more flexible temperature range can be used in the study; signal overlap does not interfere, although at least one of the exchanging resonances should be isolated; there is no need to measure $T_{1}$ or reach steady state saturation; rate constant values are measured directly and $T_{1}$ values are obtained in the same experiment, using only one set of experiments. Another remarkable advantage of the SSTD NMR methodology is that, in contrast to lineshape analysis, the determination of the kinetics rate constants is not limited by the increase in coalescence temperatures associated with high magnetic fields. Thus, our methodology is then very well appropriated for both low and high magnetic fields. This article is intended to help new users apply this new method to their own systems undergoing chemical exchange, and describes sample preparation, experimental set up, data acquisition, and an example of data processing and analysis in a simple organic molecule.

Caution: Please consult all relevant material safety data sheets (MSDS) before use.

\section{NMR Sample Preparation}

1. Weigh $5 \mathrm{mg}$ of $\mathrm{N}, \mathrm{N}$-dimethylacetamide, add to an NMR tube appropriate for low temperatures and dissolve in $0.6 \mathrm{ml}$ of toluene- $d_{8}$.

\section{NMR Experimental Setup ${ }^{25}$}

1. NOE Spectra Acquisition

1. Perform a one dimensional NOE (Nuclear Overhauser Effect) experiment. ${ }^{26}$

NOTE: NOE effects can happen at any temperature. A one dimensional NOE spectrum irradiating the signal that will be irradiated in the SSTD NMR experiment, was recorded at $-40{ }^{\circ} \mathrm{C}$ to make sure that the rotation and magnetization transfer in the sample used here was minimized, and therefore the NOE, if existent, would predominate and be measured in this experiment. Ideally, NOE effects between the two exchanging nuclei should not be present to avoid interferences with the SSTD method.

2. SSTD NMR Experiments Setup

1. Insert the sample in the magnet by first typing ej in the command line of the software to turn on the air flow. Then, put the sample on top of the magnet and then type ij. Wait until the sample is inside the magnet.

2. Once the sample is in the magnet, type edte in the command line. Change the temperature to the first selected temperature to carry out the experiment ( $295.5 \mathrm{~K}$ in this case). Let the sample stabilize at the chosen temperature for at least $20 \mathrm{~min}$.

3. Perform a $1 \mathrm{D}^{1} \mathrm{H}$-NMR experiment on the sample.

1. Create a new dataset of a ${ }^{1} \mathrm{H}-\mathrm{NMR}$ experiment. For this click on FILE/NEW and name the new experiment.

2. Type sequentially and waiting for the previous command to finish: lock, atma, topshim, getprosol and rga.

3. Type $z g$ to acquire the proton experiment. Once it is finished type efp and apk to Fourier transform it and adjust the phase.

4. Create a new dataset of, for example, a ${ }^{1} \mathrm{H}$ NMR experiment. For this click on FILE/NEW and name the new experiment.

5. In this new dataset, type rpar in the command line. Select one of the "STDDIFF" parameter sets from the list, for example STDDIFFESGP, and click "read" and then "read all" (Figure 1). Alternatively, do this by typing rpar STDDIFFESGP all. NOTE: The experiment can be performed with this pulse sequence. However, the pulse program used in our experiment was STDDIFF.

6. To select the STDDIFF pulse sequence, click the button with three dots in the PULPROG line (Figures $\mathbf{2}$ and $\mathbf{3}$ ).

7. Before carrying out the SSTD NMR experiment, calibrate the ${ }^{1} \mathrm{H} 90^{\circ}$ hard pulse $(p 1)$. For this purpose, ensure that the sample is in the magnet at the desired temperature (Step 2.2.2). Type pulsecal in the command line and copy the value of the $90^{\circ}$ pulse at the higher power ( $\mathrm{pl} 1=-1 \mathrm{~dB}$ in this case), i.e., the one that gives the shortest pulse (Figure 4).

8. Introduce the values for the calibrated hard pulse in the experiment. Type getprosol $1 \mathrm{H}$ (value for $\mathrm{p} 1$ obtained in step 2.2 .7 ) (value for pl1) (Figure 5).

9. Set the length of the shaped pulse. Type $p 13$ and introduce a value of 50,000 $\mu$ sec (Figure 6).

10. Set the selective pulse shape. To do this, go to Power and click the "Edit..." button next to SHAPE (Figure 7). Go to the shaped pulse 13 and choose: Gaus 1.1000 (Figure 8).

11. Set the selective pulse power (SP13). Set it to something appropriate, i.e., on this system between $40-60 \mathrm{~dB}$ (corresponding to a field strength of approximately $120 \mathrm{~Hz}$ ) (Figure 8). Excessive field strengths can lead to unacceptable saturation effects. ${ }^{27-29}$ NOTE: $50 \mathrm{~dB}$ was optimum in our case. Take into account that this is an attenuation scale, so the smaller the value the higher the power of the radiofrequency. As it corresponds to the saturating Gaussian cascade, which is applied for long time (several seconds), sp13 should not go below $40 \mathrm{~dB}$ (if needed, consult the instrument specifications, as long pulses at high power could damage the 
probehead). In our experience $41-61 \mathrm{~dB}$ above the attenuation of the hard ${ }^{1} \mathrm{H} 90^{\circ}$ pulse $(-1 \mathrm{~dB}$ in this work) works fine. Try to always select the highest attenuation possible leading to similar saturation level.

12. Type $n s$ and set it to 8 and type $d s$ and set it to 4 .

\section{NMR Data Acquisition and Processing ${ }^{25}$}

1. SSTD NMR Experiment Acquisition

1. Open the ${ }^{1} \mathrm{H}$ NMR experiment performed in step 2.2.3 to check where the signal that will be irradiated is. For this, search the experiment in the software browser, right click in the dataset and click "Display in a new window".

2. Move the cursor line to the center of the signal to irradiate and write down the chemical shift in ppm. Select the spectral width that will be used in the experiment.

NOTE: In this case, the signal which will be irradiated is at $2.17 \mathrm{ppm}$, and the spectral width used was $1.46 \mathrm{ppm}$. Ensure that no chemical shift correction is used or the irradiation frequency can be set incorrectly.

3. Go to the previously created SSTD NMR experiment with the setup mentioned in section 2.2 .

4. Create a list with the frequencies of irradiation. For this, type fq2list in the command line and select an existing list.

5. Edit the list of irradiation frequencies including the following data in the 3 first rows (Figure 9): Row 1. $P$ (indicates that the following data is in ppm); Row 2. Frequency of the signal to be irradiated in ppm, as measured in 3.1.1; Row 3.40 ppm (a frequency that is far from the ${ }^{1} \mathrm{H}$ signals of the compound so the irradiation in that frequency does not affect the spectra).

6. Save the list with a new name and then type fq2list in the command line and select the list just created.

7. To center the experiment on the signals under study, type $01 p$ and select as the center of the experiment the chemical shift of the signal that will be irradiated.

8. Type sw to select the spectral width ( $1.46 \mathrm{ppm}$ in this case, but any other spectral width can be chosen). NOTE: If the acquisition time obtained after changing the spectral width is too long (which will introduce more noise in the spectra) it can be adjusted by typing $A Q$ to provide the desired Free Induction Decay (FID) resolution (FIDRES, $0.25 \mathrm{~Hz}$ in this case).

9. Choose the value for the interscan relaxation delay $D 1$. Ensure that it is at least 1 to 5 times the value of the $T_{1}$ of the slowest relaxing proton.

NOTE: We set it up to $40 \mathrm{sec}$, which is the longest saturation time $(D 20)$ in the experiment. In this way all of the experiments will keep the same total "per scan" time (delay + saturation time + pulses + acquisition time).

10. Type $D 1$ and set it to $40 \mathrm{sec}$.

11. Set the first value for the saturation time by typing $D 20$ and setting it to $40 \mathrm{sec}$. Determine the receiver gain $(\mathrm{rg})$ automatically by typing rga.

12. Create the next experiment by typing iexpno. Type $D 20$ and select a saturation time of $20 \mathrm{sec}$ in this experiment. Type rga to automatically determine $\mathrm{rg}$.

13. Repeat the last step for $D 20=10,5,2.5,1.25,0.625,0.3 \mathrm{sec}$.

14. Once all the experiments are created, open the first one and in the command line type multizg and specify the number of experiments, 8 in this case (i.e., multizg 8).

2. SSTD NMR Experiment Processing

1. Open the PROCNO 1 (Process Number) from EXPNO 1 (Experiment Number) of the set (the one with the higher saturation time).

2. In the command line type $l b$ and set the value to 1.5 . NOTE: For spectra with very high signal-to-noise ratios this value could be decreased; inversely, it could be increased for noisy experiments, if the spectral resolution is not severely affected.

3. In the command line type efp and process FID \# = 1 ("on-resonance" spectrum) in PROCNO = 2 (Figure 10).

4. Correct the phase of the experiment by clicking the Interactive phase correction button and save it as a 2D experiment. Save and exit (Figure 11)

5. Type rep 1 in the command line to go to the PROCNO 1.

6. In the command line type efp and process FID \# = 2 ("off-resonance" spectrum) in PROCNO = 3 (Figure 12).

7. In the command line type .md and then rep 2 to show a multiple display window with both processed spectra: 2 (the one with the signal in the middle saturated) and 3 (the one in which the saturating pulse train was applied at $40 \mathrm{ppm}$ ) (Figure 13).

8. Click the button with a delta sign (Figure 13) to calculate the difference spectra and save it in PROCNO 4. Exit the multiple display window.

9. Select an integration range for the signal on the left (the signal in which the transfer of saturation due to the chemical exchange process will be observed). Always integrate the same region in PROCNO 3 and PROCNO 4. NOTE: The integration range used in this experiment was $2.55-2.67 \mathrm{ppm}$.

10. Once integrated, go to the "Integrals" tab in each of the experiments and copy the value of "Integral [abs]" (Figure 14).

11. Divide the integral in PROCNO 4 by the integral in PROCNO 3. That is the value of $\eta_{S S T D}$ for a saturation time of 40 sec $\left(\eta_{S S T D}=S p i n\right.$ Saturation Transfer Difference parameter). ${ }^{21}$

12. Repeat the procedure for the rest of the experiments with different saturation times.

\section{Data Analysis ${ }^{30}$}

1. Analysis of the Data to Get the Kinetic Parameters

1. Plot the obtained $\eta_{S S T D}$ values versus the saturation time. ${ }^{21}$

2. Perform an exponential fit to adjust the obtained curves to the equation

$\eta_{S S T D}=\eta_{S S T D}{ }^{M A X}(1-\exp (-\delta \cdot t))$

$\delta=$ dynamic constant 
$\eta_{S S T D}^{M A X}=\eta_{S S T D}$ at very long saturation time

$\mathrm{t}=$ time

3. Calculate the values of $\eta_{S S T D}{ }^{M A X}$ and $\delta$ and use them to calculate the values of the rate constants $(k)$ and relaxation times $\left(T_{1 A}\right)$ according to the following equations:

$\eta_{S S T D}{ }^{M A X}=\frac{k}{\left(1 / T_{1 A}+k\right)} \quad$ and $\quad \delta=1 / T_{1 A}+k$

$\mathrm{T}_{1 \mathrm{~A}}=$ longitudinal relaxation time constant of spin $\mathrm{A}$

$\mathrm{k}=$ mutual-site exchange kinetic rate constant

1. Obtain the kinetic rate constant by: $k=\eta_{S S T D} M A X \cdot \delta$

2. Eyring Plot to get the Thermodynamic Parameters

1. Plot $\ln (k / T)$ versus $1 / T(T=$ absolute temperature), using the values of the kinetic rates at different temperatures.

2. Perform a linear fit to adjust the data obtained to the Eyring equation:

$$
\begin{aligned}
& \ln \left(\frac{k}{T}\right)=\ln \left(\frac{k_{B}}{h}\right)-\frac{\Delta H^{\ddagger}}{R T}+\frac{\Delta S^{\ddagger}}{R} \\
& \Delta H^{\ddagger}=\text { entalphy of activation } \\
& \Delta S^{\ddagger}=\text { entropy of activation } \\
& h=\text { Plank's constant } \\
& \mathrm{R}=\text { gas constant } \\
& \mathrm{k}_{\mathrm{B}}=\text { Boltzmann constant } \\
& \mathrm{T}=\text { absolute temperature }
\end{aligned}
$$

3. Calculate the thermodynamic parameters $\Delta H^{\ddagger}$ and $\Delta S^{\neq}$.

4. Calculate the values for $E_{A(298)}$ and $\Delta G^{\neq}{ }_{(298)}$ using the following equations:

$$
\begin{aligned}
& E_{A(298)}=\Delta H^{\mp}+R T \\
& A G_{(298)}^{ \pm}=\Delta H^{\mp}-T \Delta S^{\mp} \\
& E_{A(298)}=\text { Activation Energy at } 298 \mathrm{~K} \\
& A G_{(298)}^{\neq}=\text {Gibbs Energy at } 298 \mathrm{~K}
\end{aligned}
$$

\section{Representative Results}

The SSTD NMR technique was applied for the calculation of the kinetic parameters in the rotation of the amide bond of $N, N$ dimethylacetamide. ${ }^{21}$ This is a simple example for which extensive data for comparison can be found in the literature. ${ }^{31}$

The hindered rotation around the amide bond, due to the partial double bond character in the resonance form, differentiates both methyl groups into two signals in the ${ }^{1} \mathrm{H}$-NMR spectra $\left(2.61\right.$ and $2.17 \mathrm{ppm}$ at $\left.22.5^{\circ} \mathrm{C}\right)$. Spin saturation of the signal of the methyl group at $2.17 \mathrm{ppm}\left(\mathrm{Me} \mathrm{B}^{\mathrm{B}}\right)$ leads to the disappearance of its signal in the ${ }^{1} \mathrm{H}$ NMR. Upon saturation of $\mathrm{Me}^{\mathrm{B}}$, transfer of saturation to the other methyl group (Me ${ }^{\mathrm{A}}$ ) due to the internal rotation process can be observed by a decrease in ${ }^{1} \mathrm{H}$ intensity in the signal at $2.61 \mathrm{ppm}$. The magnitude of this decrease will depend on the saturation time. Figure 15 shows the ${ }^{1} \mathrm{H}$ NMR spectra of the $\mathrm{N}, \mathrm{N}$-dimethylacetamide at $22.5^{\circ} \mathrm{C}$, and the expansions show the spectra without (a) and with (b) saturation of the methyl group at $2.17 \mathrm{ppm}$, as well as the difference spectrum (c), used to calculate the values of $\eta_{s s t D}$ The $\eta_{S S T D}$ factor is calculated dividing the value of the integral of Me ${ }^{A}$ in the SSTD NMR spectrum (c) by the value of the integral of the Me ${ }^{A}$ in spectra (a), as explained in the protocol. The obtained values of $\eta_{\text {SSTD }}$ for each saturation time at different temperatures are gathered in Table 1. The plot of the obtained values of $\eta_{\text {SSTD }}$ versus the saturation time gave exponential curves in which a plateau was reached at higher saturation times. For a certain temperature, the exponential fit of the curve permits the calculation of the rate constant $(k)$ and the relaxation time of the ${ }^{1} \mathrm{H}$ of the measured signal $\left(T_{1 A}\right)$ (Figure 16). Figure 17 shows all the obtained curves along with the $k$ and $T_{1 A}$ values obtained in the fits.

Finally, the plot of $\ln (k / T)$ versus $1 / T$ and the fit to the Eyring equation (Figure 18) were used to calculate the enthalpy and entropy of activation. The determined activation parameters are shown in Table 2, together with the previously reported parameters calculated using different methodologies.

As can be observed in Table 2, the values of the activation parameters obtained with the Spin Saturation Transfer Difference technique (SSTD NMR) are in excellent agreement with the data previously reported using other techniques, such as SST NMR or line shape analysis. The wide range of values reported for $\Delta S^{\ddagger}$ is due to the difficulty in the measurement of this parameter with NMR techniques. ${ }^{31}$ As for the rest of the activation parameters, the values obtained with our method are not only really similar to the ones already reported but also more accurate, since our errors (SD) are smaller in all the cases. 


\begin{tabular}{|c|c|c|c|c|c|}
\hline () & & Parameter Sets: rpar & & & $x$ \\
\hline Eile options Help & & & Source $=/$ opt & pspin/exp/stan/nmr/par & 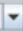 \\
\hline Search in names $\left[{ }^{*} ?\right]=$ Se & arch & & & & \\
\hline & 1 & & & & \\
\hline NA_HSQCETF 3CPXY & NA_HSQCETCPJCSP & NA_HSQCF3CPJRPHXY & NA_HSQCF3GPNOPHXY & NA_NOESYCPPHPRXF & \\
\hline NA. NOESYCPPHXF 19 & NA_NOESYHSQCCTET CP $3 D$ & NA_NOESYHSQCF3CPWC3D & NA.PCCHCO & NA, PCCHDI & \\
\hline NA_PCCHDI2 & NA_PFIDSETCPSI & NA_TRHCCHCO3D & NA_TRHCNCHMQSP3D & NA_TRHCNCHMQSPWC3D & \\
\hline NA_TRHCNCHMQSPWC3D2 & NA_TRHCNET & NA_TRHCNETSI3D & NA_TRHCIPH & NA_TRHCNPH3D & \\
\hline NA_TRHNNCOSYCPPHSPWC & NA, X OHCOCTETF 3 CP & NA XHCOETF $3 G P$ & NOEDIFF & NOESYCNCPSD & \\
\hline NOESYESCPPH & NOESYF3CPPHXF 19 & NOESYCPPH19SW & NOESYCPPHWCXX1 & NOESYCPPHWCX2 & \\
\hline NOESYCPPHWWXF & NOESYCPPHWCXF.2 & NOESYHSOCEDCPSISMBD & NOESYHSOCEDCPSM $3 D$ & NOESYHSOCETIF 3CP3D & \\
\hline NOESYHSQCET CP3D & NOESYHSQCF3CP193D & NOESYHSQCF3CPSI3D & NOESYHSQCF 3 CPWCX $13 D$ & NOESYHSQCFPF 3 CPSI3D & \\
\hline NOESYHSQCCPSISMSP3D & NOESYHSQCCPSM3D.2 & NOESYHSQCCPWCX $13 D$ & NOESYNCCP3D & NOESYPHPR & \\
\hline NOESYPHSW & NOESYPR1D & NOESYTRETF 3 CP $3 D$ & NOESYTZGP3D & $0172 \mathrm{C}$ & \\
\hline OICALIBRATION & P31 & P31CPD & P3919GP & PROBIIDEC & \\
\hline PROF 19DEC & $\mathrm{PROF}_{2} 1 \mathrm{H}$ & PROF_1H_PLASMA & PROF_COSY & PROF_CPMC & \\
\hline PROF_DIFF & PROF_JRES & PROHOMODEC & PROHUMP & PRONRLFEXP & \\
\hline PROP31DEC & PRORESOL & PROSENS & PROT-VT & PROTON & \\
\hline PROTON128 & PROTON256 & PROTONCONLF & PROTONEAP & PROTONLF & \\
\hline PROTONLFEP & PROTONNR & PROTONINREXP & PROTONINRLF & PROTONT1 & \\
\hline PROTONInfo & PT195ZC & RH103ZC & ROESYPHPR & ROESYPHSW & \\
\hline SE77ZC & SELCOIH & SELCOCP & SELGPSE & SEIMLCP & \\
\hline SEMMLZF $1 \mathrm{H}$ & SEUNO1H & SEUNOCP & SELRO1H & SELROCP & \\
\hline SELZC1H & SEQTRCBCANHCP3D & SEQTRHNCACBET CP3D & SEQTRHNCACBCP3D & SEQTRHNCAET CP3D & \\
\hline SEQTRHNCACP3D & SFHMQCF3CPPH & SI291C & SN119IC & STDDIFFESCP & \\
\hline STDDIFFESCP. 2 & STDDIFFESCP.3 & STDOIFFCP 19 & STDDIFFCP19.2 & STDDIFFCP 19.3 & \\
\hline STDHSQCET CPSISP & STDMLEVESCPPH & STDNOESYESCPPH & TRCBCACONHCP3D & TRCBCANHET CP3D & \\
\hline TRCBCANHCP 30 & TRHBHACONHET CP3D & TRHEHACONHCP3D & TRHBHANHETCP3D & TRHEHANHCP3D & \\
\hline TRHNCACBCP2H3D & TRHNCACBCP $3 D$ & TRHINCACBIETCPBD & TRHNCACBICP $3 D$ & TRHNCACOETCP3D & \\
\hline TRHINCACOCP2H3D & TRHNCACOCP3D & TRHNCAETCP3D & TRHNCACP2H3D & TRHNCACP2H3D2 & \\
\hline TRHINCACP3D & TRHNCACP 3D. 2 & TRHINCACP3D2 & TRHNCACP3D2.2 & TRHNCAIETCP3D & \\
\hline TRHNCAICP3D & TRHNCOCACBET CP 3D & TRHNCOCACBCP2H3D & TRHNCOCACBCP3D & TRHNCOCAET CP3D & \\
\hline TRHINCOCACP2H3D & TRHNCOCACP3D & TRHNCOETCP3D & TRHNCOCP2H3D & TRHNCOCP3D & \\
\hline TRHNCOCPRC $3 D 1$ & TRHNCOCPRC 302 & TRHNCOCPRC3D4 & TRHINCOCPRC 305 & TRHNCOCPRC3D6 & \\
\hline TRNOEF 3CPSI & TROSYARCPPHWG & TROSYETF 3 CPSI & TROSYF 3 CPPH 19 & TROSYF3CPPHSI 19 & \\
\hline TROSYF 3CPPHSI 19.2 & TRT1ETF3CPSI & TRT2ETF3CPSI & TopCuide 1D & Tune2h & \\
\hline Tunes & Tunef & Tuneh & Tunep & WATER & \\
\hline WATERSUP & XCTB & ZCCPPR & ZGESCP & ZCCPWC & \\
\hline ZCPR & gradshimld1h & gradshim1d2h & gradshimdata & gradshimrcb3d & \\
\hline standard 10 & standard $2 \mathrm{D}$ & standard3D & topshim1d1h & topshim1d2h & \\
\hline topshim3d1h & & & & & $\nabla$ \\
\hline & & & & Read... & \\
\hline
\end{tabular}

Figure 1: List of experiments after typing rpar. The figure shows the different parameter sets among which STDDIFFESGP should be selected. Please click here to view a larger version of this figure.

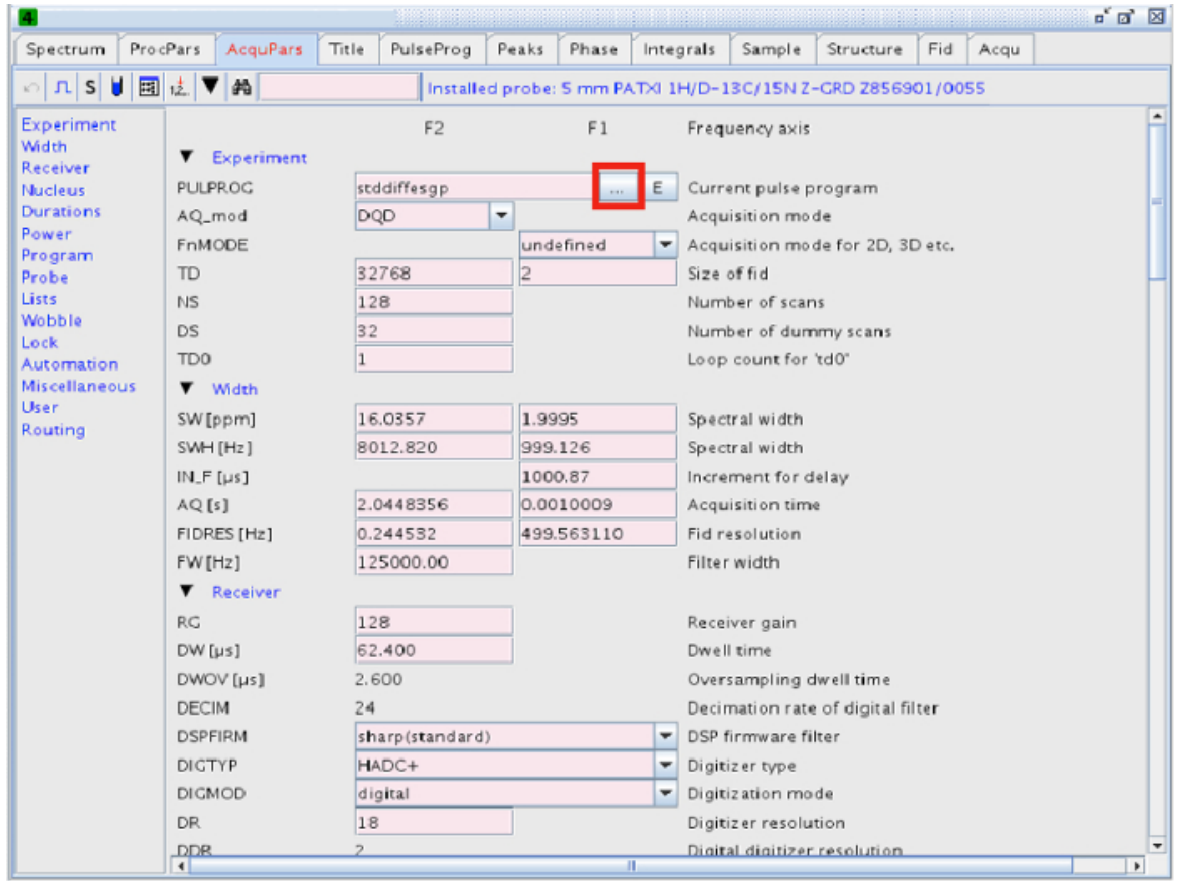

Figure 2: Acquisition parameters. The button highlighted in a red square leads to a list of the different pulse programs. Please click here to view a larger version of this figure. 


\begin{tabular}{|c|c|c|c|c|c|}
\hline LI & & Pulse Programs & & & $\bar{x}$ \\
\hline Eile Options Help & & & Source $=10 \mathrm{pt} /$ & n/exp/stan/nmr/lists/pp & 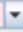 \\
\hline Search in names $\left.\left[{ }^{\circ}\right]\right]=$ & Search & & & & \\
\hline Class $=$ Any & - $\operatorname{Dim}=\operatorname{Any}=$ & & & & \\
\hline All & & & & & \\
\hline & & & & & \\
\hline scosyph & scosyphrd & secsyiraf & secsyaf & selco & - \\
\hline selcogp & selcopg & selcorl & seldigp & selgpse & \\
\hline selhsqcgpirndsp & selhsqcgpsisp & selina & selineptirrdsp & seljresqfsp & \\
\hline selmlgp & selmigp.2 & selmizf & selno & selnogp & \\
\hline selnogpzs & selnozf & selro & selrogp & selrogp.2 & \\
\hline selzg & selzgpg & sequrcbcanhgp $3 d$ & sequtrhncacbetgp $2 \mathrm{~h} 3 \mathrm{~d}$ & sequthncacbetgp $3 \mathrm{~d}$ & \\
\hline seqtrhncacbgp $2 h 3 d$ & seqtrhncacbgp $3 d$ & sequthncaetgp $2 \mathrm{~h} 3 \mathrm{~d}$ & sequtrhncaetgp $3 d$ & seqtrhncagp $2 \mathrm{~h} 3 \mathrm{~d}$ & \\
\hline seqtrhncagp 3d & sfhmgcf3gpph & shmbcgpndqf & stddiff & stddiff.2 & \\
\hline stddiff. 3 & stddiffesgp & stddiffesgp.2 & stddiffesgp. 3 & stddiffgp 19 & \\
\hline stddiffgp 19.2 & stddiffgp 19.3 & stdhsqcetgpsisp & stdhsqcetgpsp & stdmlevesgpph & \\
\hline stdmlevgpph19 & stdmlevph & stdnoesyesgpph & stdnoesyesgpph.2 & stdnoesygpph & \\
\hline stdnoesygpph.2 & stdnoesygpph19 & stdnoesygpph19.2 & stebpgp $1 \mathrm{~s}$ & stebpgp 1s19 & \\
\hline stebpgp 1s191d & stebpgp1s1d & stebpgpin $1 \mathrm{~s}$ & stebpgpin1s1d & stegp is & \\
\hline stegp 1s1d & sys 13 deg & sys 13 dsp & syscancel & sysdante & \\
\hline sysdecpro & sysdrift & sysglitch & sysgrcan & sysp lincr & \\
\hline sysp 3 incer & sysphas $2 \mathrm{f} 1$ & sysphasf1 & syspreempgp & sysselzg2d & \\
\hline sysselzg 4 & sysselzg5 5 & systurnon & syszg2d & syszged.2 & \\
\hline syszg2df2gppr & syszg2df2pr & syzzg 4 & syszggegp $2 d$ & syszggp $2 d$ & \\
\hline syszgr & tlir & thir1d & rlirpg & topshim1d & \\
\hline ropshim3d & trebcaconhetgp $3 d$ & trcbcaconhgp $3 d$ & ercbcanhetgp $3 d$ & trcbcanhgp $3 d$ & \\
\hline trhbhaconhetgp 3d & trhbhaconhgp 3d & trhbhanhetgp 3d & trhbhanhgp $3 d$ & trhocconhetgp $3 \mathrm{~d} 2$ & \\
\hline trhccconhetgp $3 \mathrm{~d} 3$ & trhccconhetgp $3 \mathrm{~d} 3.2$ & trhecconhgp $3 \mathrm{~d} 2$ & trhecconhgp $3 \mathrm{~d} 3$ & trhecconhgp $3 \mathrm{~d} 3.2$ & \\
\hline trhecheogp3d & trhncacbetgp $2 \mathrm{~h} 3 \mathrm{~d}$ & trhncacbetgp $3 d$ & trinncacbgp $2 \mathrm{~h} 3 \mathrm{~d}$ & trhncacbgp $3 d$ & \\
\hline uhncacbietgp $2 h 3 d$ & thnncacbietgp 3d & trhncacbigp $2 \mathrm{~h} 3 \mathrm{~d}$ & trhncacbigp $3 \mathrm{~d}$ & trhncacoetgp $2 \mathrm{~h} 3 \mathrm{~d}$ & \\
\hline trhncacoetgp $3 d$ & thhncacogp $2 h 3 d$ & trhncacogp3d & trhncaetgp $2 \mathrm{~h} 3 \mathrm{~d}$ & trhncaetgp 3d & \\
\hline trinncagp $2 \mathrm{~h} 3 \mathrm{~d}$ & trhncagp $2 \mathrm{~h} 3 \mathrm{~d} 2$ & trhncagp $3 d$ & trhncagp 3d.2 & trhncagp 3d2 & \\
\hline trhncagp $3 \mathrm{~d} 2.2$ & trhncagprc3d1 & trhncaietgp $2 h 3 d$ & trhncaietgp 3d & trhncaigp $2 \mathrm{~h} 3 \mathrm{~d}$ & \\
\hline trhncaigp 3d & thincocacbetgp $2 \mathrm{~h} 3 \mathrm{~d}$ & trhncocacbetgp 3 d & trhncocacbgp $2 h 3 d$ & trhnncocacbgp $3 d$ & \\
\hline trhncocaetgp $2 \mathrm{~h} 3 \mathrm{~d}$ & trhncocaetgp $3 d$ & trhncocagp $2 \mathrm{~h} 3 \mathrm{~d}$ & trhncocagp 3d & trhncoetgp3d & \\
\hline trhncogp $2 h 3 d$ & trhncogp $3 d$ & trhncogphb3d & erhncogpre $3 \mathrm{~d} 1$ & trhncogpre3d2 & \\
\hline trhncogpre3d3 & trhneogpre3d4 & trhncogpre3d5 & trhncogpre3d6 & trhncogpre3d7 & \\
\hline thincorexf3gp & tohncot1f3gp & trhncot2f3gp & urhncotrf3gp & trnoef3gpsi & \\
\hline troesuph & troesvphpr & trosvarapphwg & trosycxf3apphsi19 & trosverf3 apiasi & - \\
\hline & & & & \begin{tabular}{|l|l|} 
Qk & Sancel \\
\end{tabular} & \\
\hline
\end{tabular}

Figure 3: List of pulse programs. The figure shows the selected pulse program in the experiment (STDDIFF). Please click here to view a larger version of this figure.

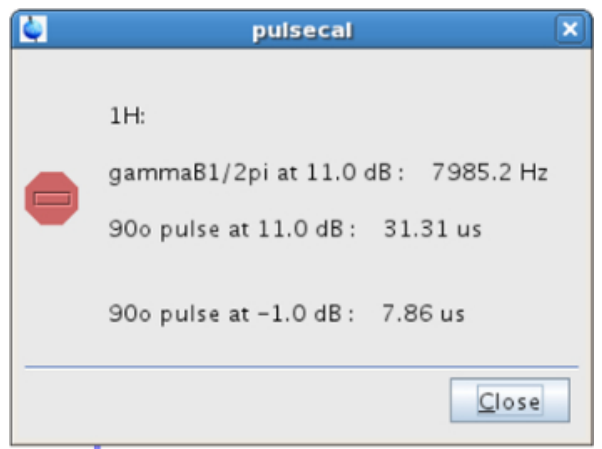

Figure 4: Pop-up window appeared after the $90^{\circ}$ pulse calibration. The figure shows the values of the calibrated $90^{\circ}$ pulse at different power levels. Please click here to view a larger version of this figure.

\section{getprosol $1 \mathrm{H} 7.86-1.0$}

Figure 5: Screenshot of the command line. The figure shows how to introduce the value for the calibrated hard pulse. Please click here to view a larger version of this figure.

\begin{tabular}{|c|c|c|}
\hline ל. & P1 & $x$ \\
\hline \multicolumn{3}{|c|}{ Pulse } \\
\hline \multicolumn{3}{|c|}{ P13 $[\mu \mathrm{s}] 50000.00$} \\
\hline & 으 & Cancel \\
\hline
\end{tabular}

Figure 6: Value for the length of the shaped pulse. The figure shows how to introduce the value for the length of the shaped pulse. Please click here to view a larger version of this figure. 


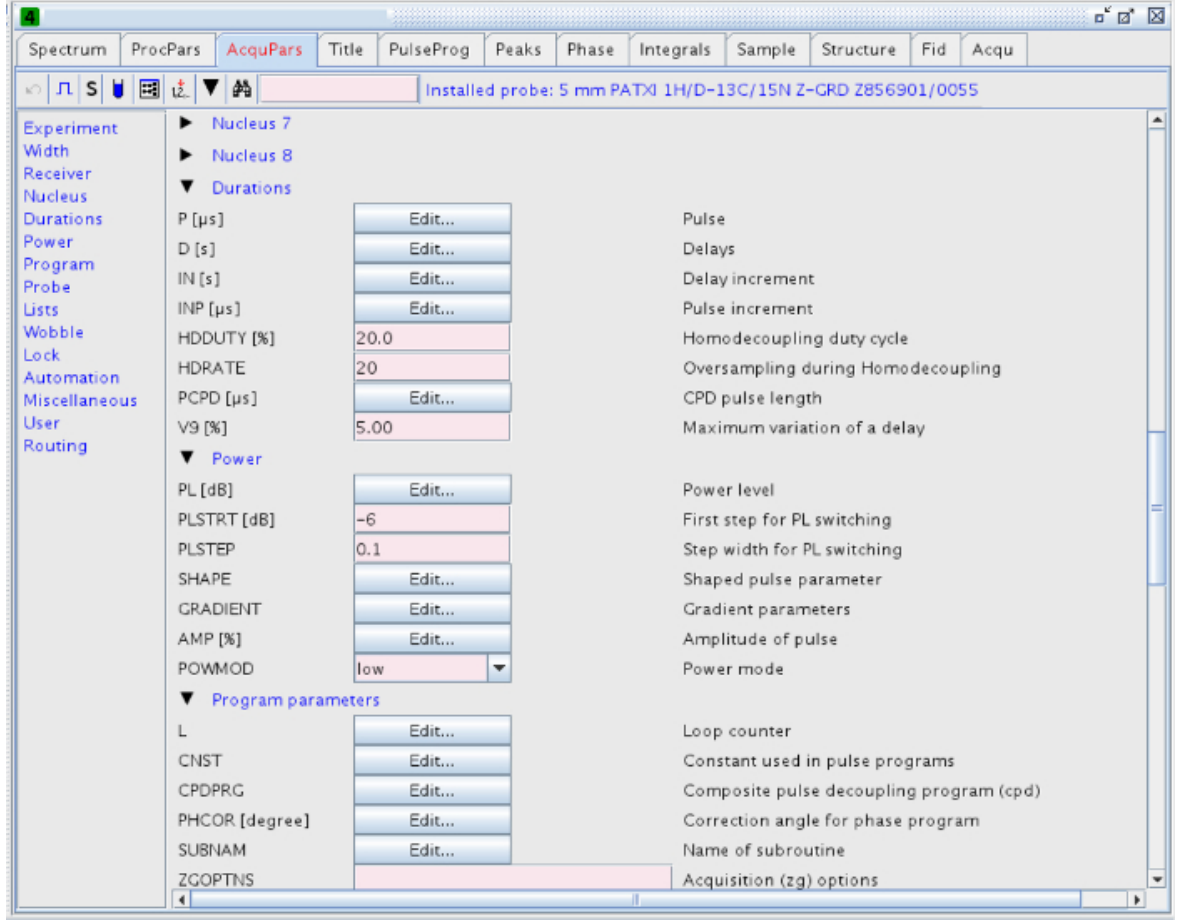

Figure 7: Acquisition parameters. The figure shows the power parameters. Please click here to view a larger version of this figure.

\begin{tabular}{|c|c|c|c|c|c|c|c|}
\hline हा & & & dit... & & & & $x$ \\
\hline Shaped & pulse parameter & & & & & & \\
\hline Index & $\begin{array}{c}\text { Power }[\mathrm{dB}] \\
\text { (SP) }\end{array}$ & $\begin{array}{c}\text { Offset-Freq. [Hz] } \\
\text { (SPOFFS) }\end{array}$ & $\begin{array}{c}\text { Phase alignment } \\
\text { (SPOAL) }\end{array}$ & $\begin{array}{l}\text { Filename } \\
\text { (SPNAM) }\end{array}$ & & & \\
\hline 0 & 1 & 0 & 0.5 & gauss & $\ldots$ & $E$ & $\Delta$ \\
\hline 1 & 43.4 & 0 & 0.5 & Squa 100.1000 & $\ldots$ & $E$ & \\
\hline 2 & 73.18 & 0 & 0.5 & Gaus1_180r.1000 & $\ldots$ & $\mathrm{E}$ & \\
\hline 3 & 2.44 & 0 & 0.5 & Crp60,0.5,20.1 & $\ldots$ & $E$ & \\
\hline 4 & 0 & 0 & 0.5 & gauss & $\ldots$ & $E$ & \\
\hline 5 & 0 & 0 & 0.5 & gauss & $\ldots$ & $E$ & \\
\hline 6 & 68.85 & 0 & 0.5 & Squa 100.1000 & $\ldots$ & $E$ & \\
\hline 7 & 2.44 & 0 & 0.5 & Crp60comp.4 & $\ldots$ & $E$ & \\
\hline 8 & 0 & 0 & 0.5 & gauss & $\ldots$ & $\mathrm{E}$ & \\
\hline 9 & 0 & 0 & 0.5 & gauss & $\ldots$ & $\mathrm{E}$ & \\
\hline 10 & 0 & 0 & 0.5 & gauss & $\ldots$ & $E$ & \\
\hline 11 & 0 & 0 & 0.5 & gauss & $\ldots$ & $\mathrm{E}$ & \\
\hline 12 & 0 & 0 & 0.5 & gauss & $\ldots$ & $\mathrm{E}$ & \\
\hline 13 & 50 & 0 & 0.5 & Gaus 1.1000 & $\ldots$ & $E$ & \\
\hline 14 & 2.95 & 0 & 0.5 & Crp32,1.5,20.2 & $\ldots$ & $\mathrm{E}$ & \\
\hline 15 & 0 & 0 & 0.5 & gauss & $\ldots$ & $E$ & \\
\hline 16 & 150 & 0 & 0.5 & gauss & $\ldots$ & $E$ & \\
\hline 17 & 150 & 0 & 0.5 & gauss & $\ldots$ & $E$ & v \\
\hline & & & & ㅇK & & ance & \\
\hline
\end{tabular}

Figure 8: Parameters for the shaped pulse. The values for the shaped pulse will be introduced in line 13. Please click here to view a larger version of this figure. 


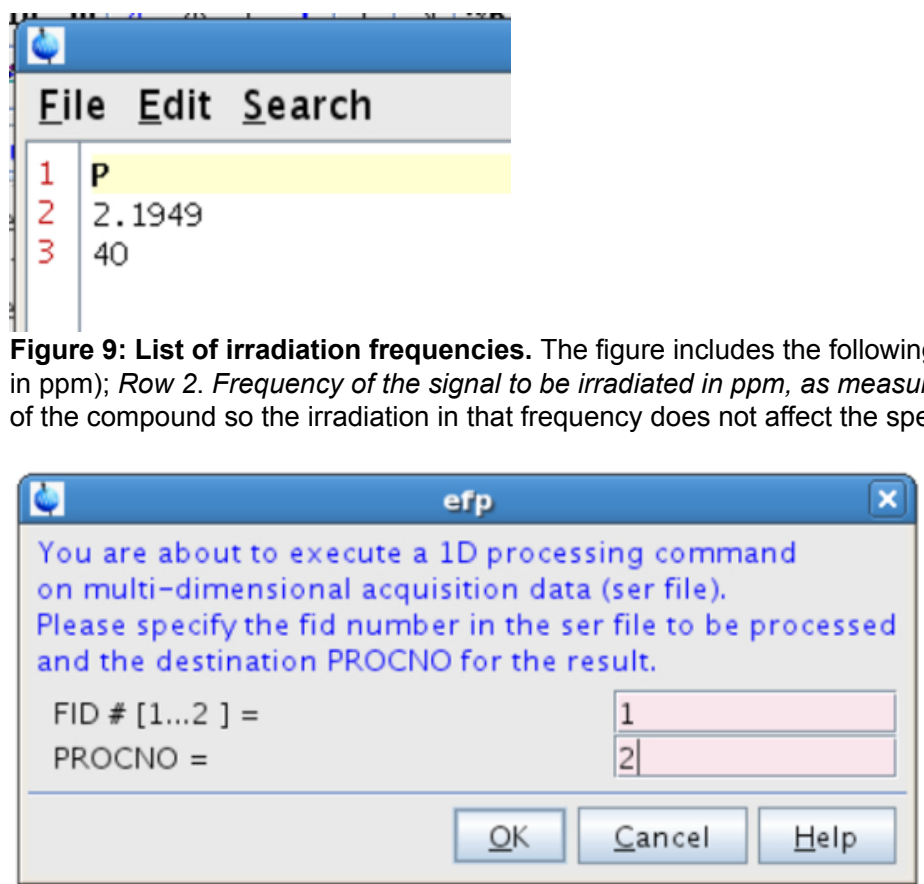

Figure 10: Processing of the first FID. The figure shows the pop up window that appears after typing efp. Please click here to view a larger version of this figure.

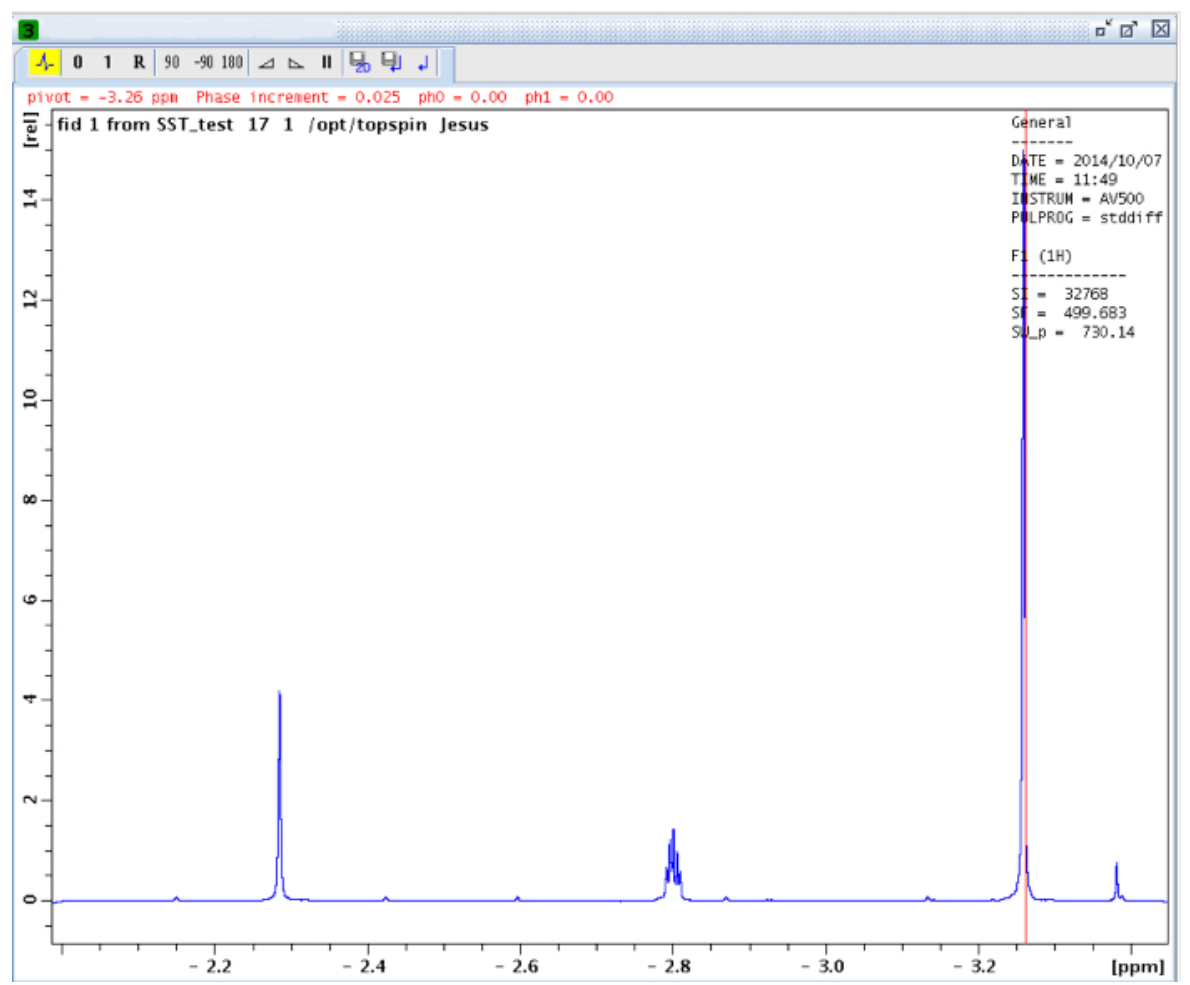

Figure 11: Phase correction. Screenshot showing the window for the manual phase correction. Please click here to view a larger version of this figure. 


\begin{tabular}{l}
\hline efp \\
You are about to execute a 1D processing command \\
on multi-dimensional acquisition data (ser file). \\
Please specify the fid number in the ser file to be processed \\
and the destination PROCNO for the result. \\
FID \#[1...2 $]=$ \\
PROCNO = \\
\hline$\underline{\text { OK }}$ Cancel \\
\hline
\end{tabular}

Figure 12: Processing of the second FID. The figure shows the pop up window that appears after typing efp. Please click here to view a larger version of this figure.

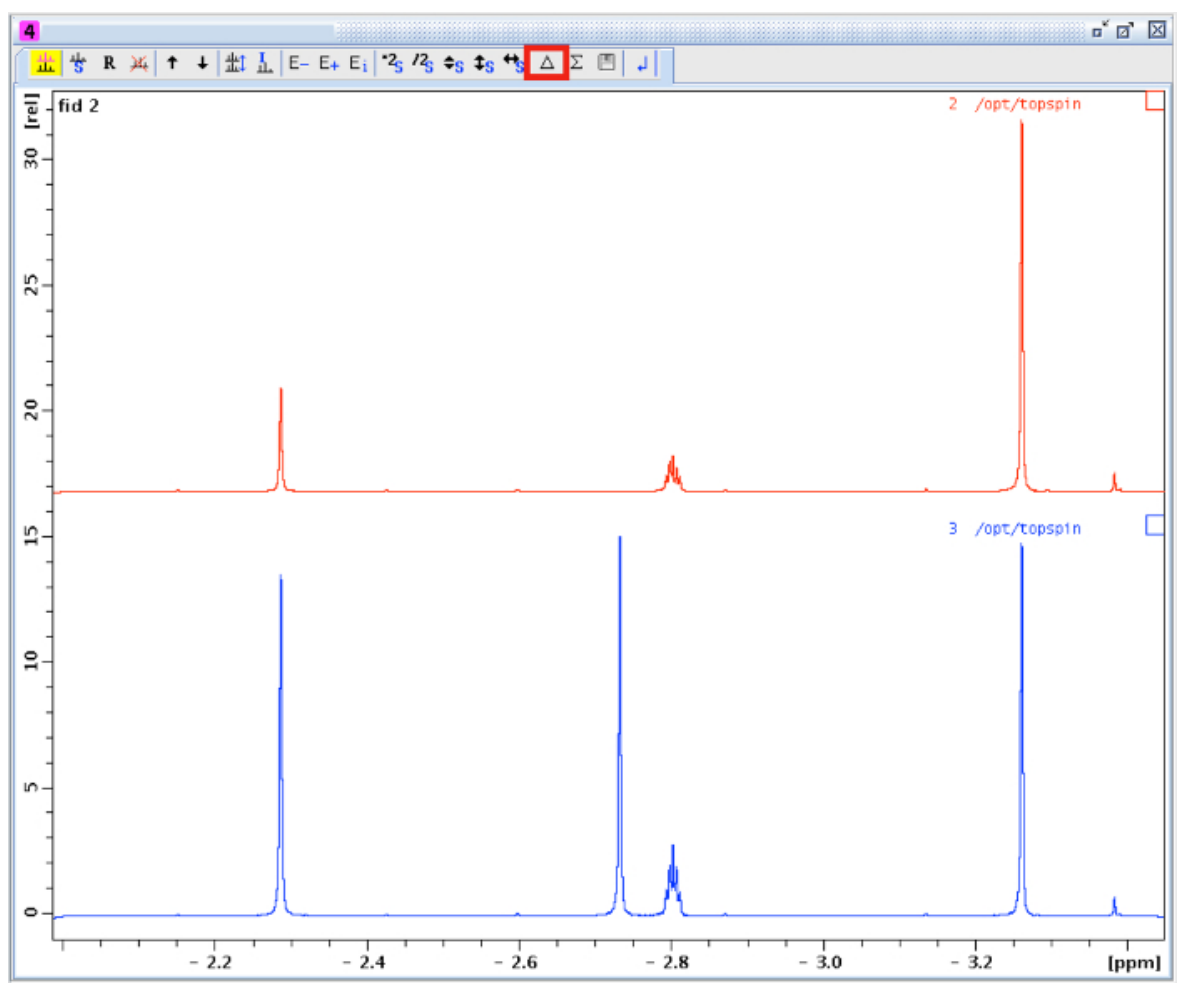

Figure 13: Multiple display of spectra 2 and 3. The button highlighted in a red square is the one to calculate the difference spectra. Please click here to view a larger version of this figure.

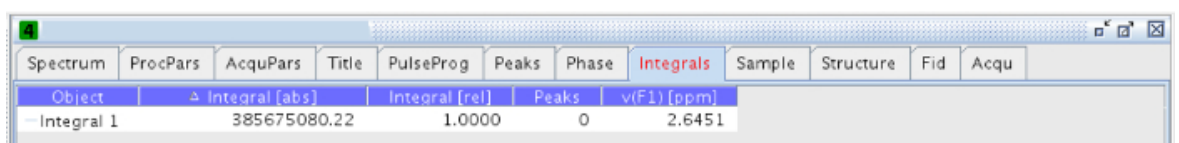

Figure 14: Integrals tab. The figure shows the values of the absolute and relative integrals. Please click here to view a larger version of this figure. 


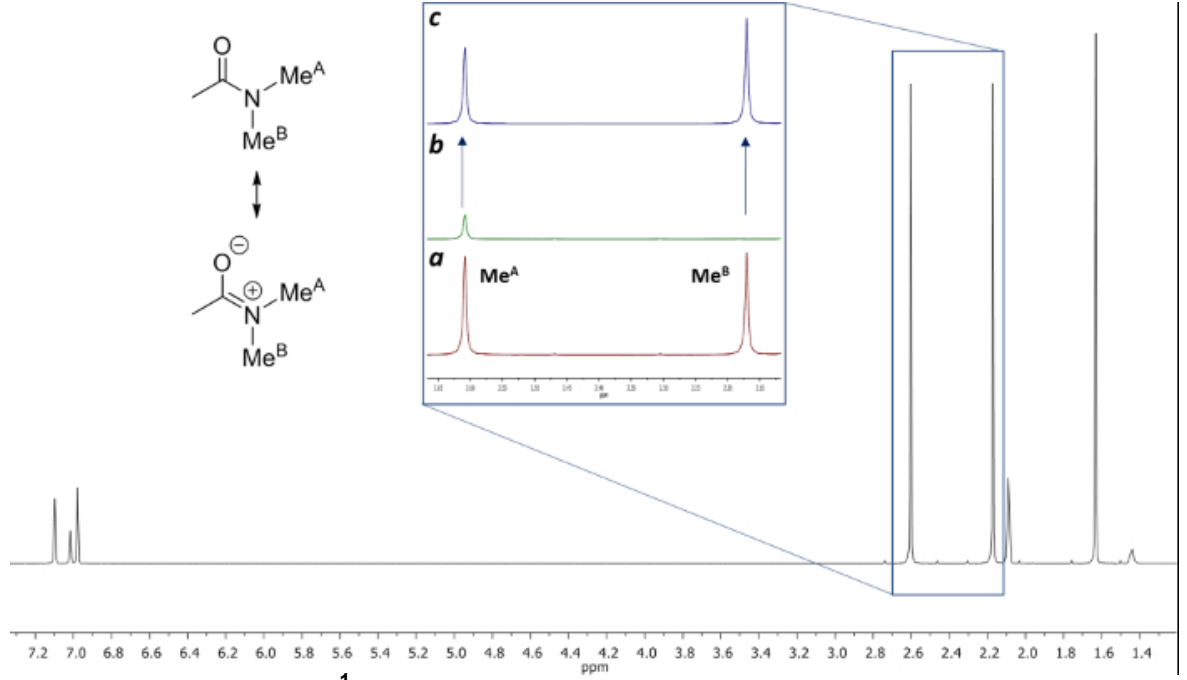

Figure 15: Structure and ${ }^{1} \mathrm{H}$ NMR spectra of $\mathbf{N}, \mathbf{N}$-dimethylacetamide at $22.5^{\circ} \mathrm{C}$ in toluene-d $\mathrm{d}_{8}$. (a) ${ }^{1} \mathrm{H}$ NMR expansion of the region from 2.13 to $2.66 \mathrm{ppm}$ before irradiation. (b) Expansion of the same region after irradiation of the methyl group at $2.17 \mathrm{ppm}$. (c) Difference spectrum [(a)(b)]. Please click here to view a larger version of this figure.

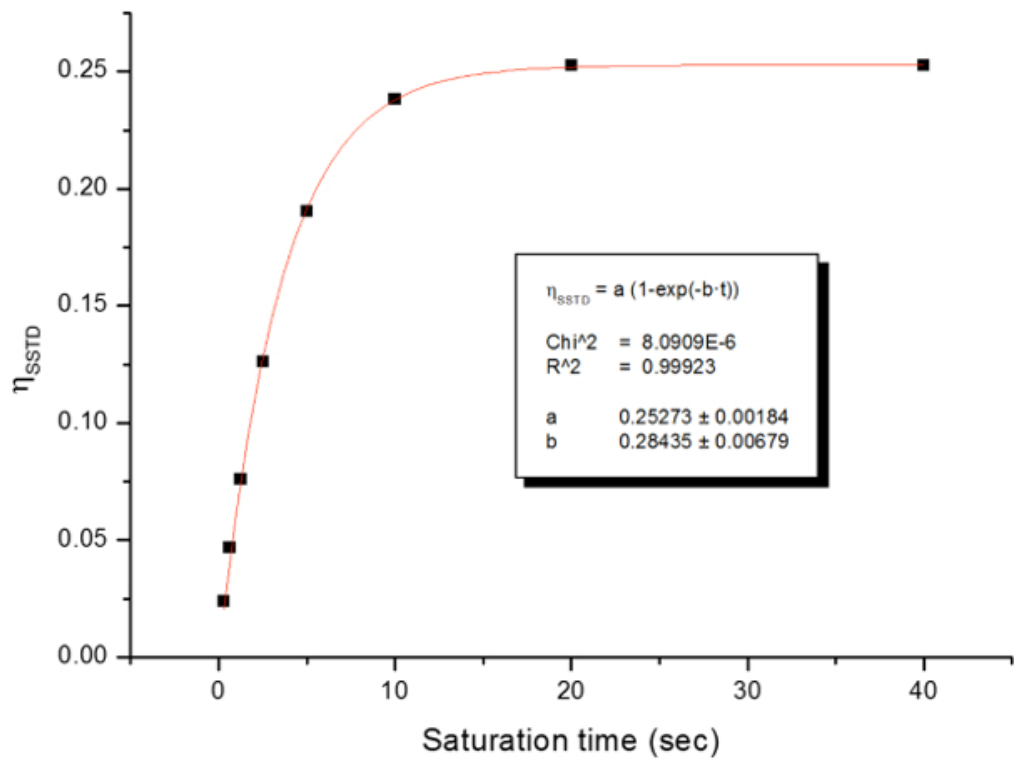

Figure 16: Example of the plot of $\eta_{S S T D}$ and its exponential fit at $278 \mathrm{~K}$. Reproduced from the supporting information of reference ${ }^{21}$ with permission from the Royal Society of Chemistry. Please click here to view a larger version of this figure. 


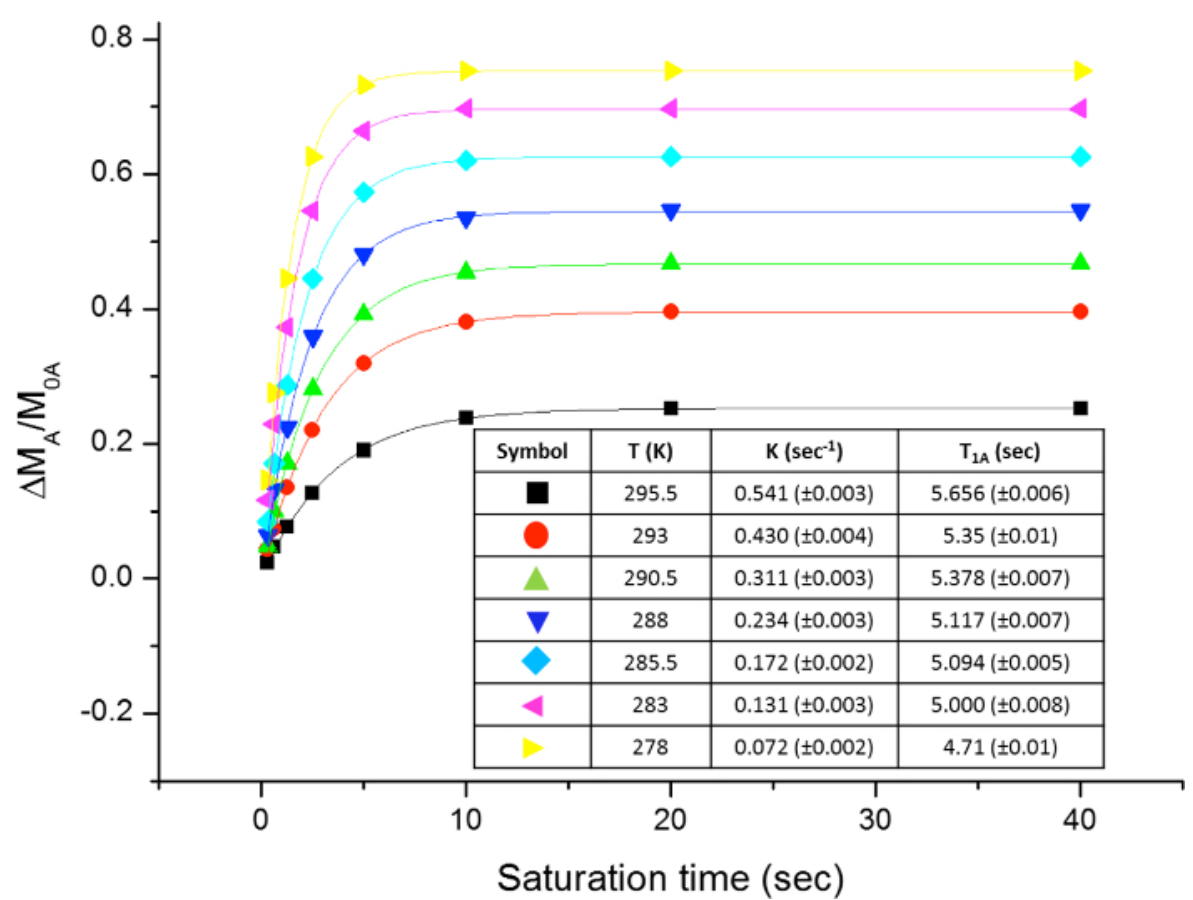

Figure 17: Plots of $\eta_{\text {SSTD }}$ vs. saturation time at different temperatures. The figure shows the plot for $N, N$-dimethylacetamide and the table with the obtained rates constants and relaxation times. Please click here to view a larger version of this figure.

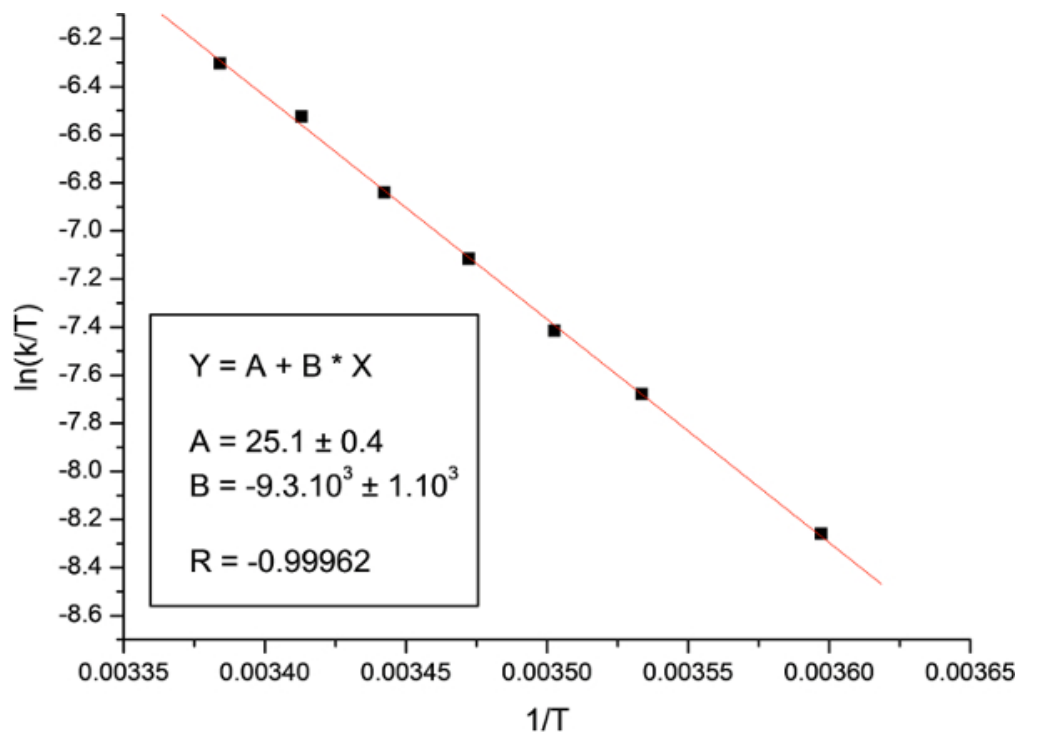

Figure 18: Eyring plot. The figure shows the plot for $\mathrm{N}, \mathrm{N}$-dimethylacetamide. Please click here to view a larger version of this figure. 


\begin{tabular}{|c|c|c|c|c|c|c|c|}
\hline \multirow[t]{2}{*}{$t_{\text {sat }}(\mathrm{sec})$} & $\eta_{\text {SSTD }}$ & $\eta_{\text {SSTD }}$ & $\eta_{\text {SSTD }}$ & $\eta_{\text {SSTD }}$ & $\eta_{\text {SSTD }}$ & $\eta_{\text {SSTD }}$ & $\eta_{\text {SSTD }}$ \\
\hline & $(\mathrm{T}=278 \mathrm{~K})$ & $(\mathrm{T}=283 \mathrm{~K})$ & $(\mathrm{T}=285.5 \mathrm{~K})$ & $(\mathrm{T}=288 \mathrm{~K})$ & $(T=290.5 \mathrm{~K})$ & $(T=293 \mathrm{~K})$ & $(\mathrm{T}=295.5 \mathrm{~K})$ \\
\hline 40 & 0.2526 & 0.3957 & 0.4671 & 0.5461 & 0.626 & 0.6969 & 0.7535 \\
\hline 20 & 0.2526 & 0.3957 & 0.4671 & 0.5461 & 0.626 & 0.6969 & 0.7535 \\
\hline 10 & 0.2383 & 0.3806 & 0.4537 & 0.5355 & 0.6199 & 0.6969 & 0.7535 \\
\hline 5 & 0.1904 & 0.3193 & 0.3919 & 0.481 & 0.5734 & 0.6638 & 0.7318 \\
\hline 2.5 & 0.1263 & 0.2204 & 0.2812 & 0.3589 & 0.4449 & 0.5461 & 0.626 \\
\hline 1.25 & 0.0761 & 0.1353 & 0.171 & 0.2247 & 0.2868 & 0.3732 & 0.4449 \\
\hline 0.625 & 0.0467 & 0.0739 & 0.099 & 0.1327 & 0.171 & 0.2291 & 0.2758 \\
\hline 0.3 & 0.0238 & 0.044 & 0.0472 & 0.0644 & 0.0847 & 0.1169 & 0.1463 \\
\hline
\end{tabular}

Table 1: Values of $\eta_{\text {SSTD. }}$ The table shows the values obtained at different saturation times for $N, N$-dimethylacetamide in the range of temperatures $278-295.5 \mathrm{~K}$.

\begin{tabular}{|c|c|c|c|c|c|}
\hline Method & SSTD NMR & \multirow[t]{2}{*}{ SST NMR ${ }^{31}$} & Line-shape analysis & Line-shape analysis & Line-shape analysis \\
\hline Parameter & (This work) & & $\left({ }^{1} \mathrm{H} \text { NMR }\right)^{4}$ & $\left({ }^{1} \mathrm{H} \text { NMR }\right)^{5}$ & $\left({ }^{13} \mathrm{C} \text { NMR }\right)^{6}$ \\
\hline $\mathrm{E}_{\mathrm{a} 298}\left(\mathrm{KJ} \mathrm{mol}^{-1}\right)$ & $79.7 \pm 0.1$ & $73.1 \pm 1.4$ & $70.5 \pm 1.7$ & $82.0 \pm 1.3$ & $79.5 \pm 0.4$ \\
\hline$\Delta \mathrm{H}^{\ddagger}\left(\mathrm{KJ} \mathrm{mol}^{-1}\right)$ & $77.2 \pm 0.1$ & $70.6 \pm 1.4$ & 68 & $79.5 \pm 0.4$ & $76.6 \pm 0.4$ \\
\hline$\Delta \mathrm{S}^{\ddagger}\left(\mathrm{J} \mathrm{mol}{ }^{-1} \mathrm{~K}^{-1}\right)$ & $11.5 \pm 0.4$ & $-10.5 \pm 5.0$ & $-15.0 \pm 5.1$ & $13 \pm 8$ & $3 \pm 4$ \\
\hline$\Delta \mathrm{G}^{\ddagger}{ }_{298}\left(\mathrm{KJ} \mathrm{mol}^{-1}\right)$ & $73.8 \pm 0.1$ & $73.7 \pm 2.0$ & 72.5 & $75.3 \pm 0.4$ & $75.7 \pm 0.4$ \\
\hline Solvent & Tol- $d_{8}$ & Tol- $d_{8}$ & $\mathrm{CCl}_{4}$ & Acetone- $d_{6}$ & neat \\
\hline
\end{tabular}

Table 2: Activation parameters. The table shows the activation parameters for the internal rotation of $N, N$-dimethylacetamide obtained by the SSTD NMR method compared with the same parameters obtained using different NMR methods for analysis. ${ }^{4,5}$ Errors in this table refer to standard deviation errors (SD). (Reproduced from reference ${ }^{21}$ with permission from the Royal Society of Chemistry).

\section{Discussion}

One of the more obvious advantages of this methodology is that the rate constants and the relaxation time for a given temperature can be obtained with a single set of experiments, with a robust pulse sequence (the same used for STD experiments to study protein-ligand interactions, which is typically found within the available set of experiments from the spectrometer manufacturer). This simplifies the experimental setup since there is no need to measure $T_{1}$ or reach steady state saturation. Besides, it is remarkable that this method does not depend on the magnet strength, as coalescence methods. On the other hand, the main limitation is that this technique cannot be applied to chemical exchange processes too fast or too slow, which would depend on the temperature range of the NMR machine or the solvents used.

This new technique for the calculation of kinetic parameters can be applied to a great variety of substrates and its applicability has already been demonstrated with some interesting molecules. ${ }^{21}$ The kinetic parameters of the 4- $N, N$-dimethylamido[2.2]paracyclophane, a challenging substrate in which the signal of one of the methyl groups of interest is overlapped with other signals from the molecule, were successfully calculated using SSTD NMR. Interestingly, this methodology can be applied as long as one of the signals of study is isolated. SSTD NMR is also a useful protocol for the calculation of kinetic parameters in molecules in which the coalescence temperature is so high that the molecule decomposes before reaching it. This is the case with $\mathrm{PtCl}_{2}$ (dimethylallene)(pyridine), in which the methodology was successfully applied without the need of reaching coalescence. The choice of solvents and temperatures is critical to obtain good results, since the chemical exchange rates can vary significantly with these parameters. Moreover, in addition to the criteria in a normal NMR experiment, key steps in a SSTD NMR experiment are the selectivity of the irradiation as well as the temperature control. Both factors have to be precise to guarantee the success of the experiment.

The representative results presented here are for the kinetics of intramolecular chemical exchange, but the technique can also be applied to study the kinetics of intermolecular chemical exchange and also ligand exchange, common processes in the dynamic behavior of transition metal complexes.

Finally, providing a proper modification of the equations is made,$^{32}$ this method could be extended to deal with multi-site exchange and unequal populations, as it has been done in former double resonance experiments, ${ }^{8-9}$ increasing the usefulness of this technique for the study of chemical exchange processes in challenging compounds.

\section{Disclosures}

The authors declare that they have no competing financial interests. 


\section{Acknowledgements}

Funding by the University of East Anglia, the EPSRC (EP/L012855/1) and the EU (H2020-MSCA-IF-2014-EF-ST-658172) is gratefully acknowledged (MTQ).

\section{References}

1. Bain, A. D. Chemical Exchange in NMR. Prog. Nuc. Mag. Res. Spect. 43, 63-103 (2003).

2. Bain, A. D. Chemical Exchange. Modern Magnetic Resonance. 421-427 (2006).

3. Bain, A. D. Chapter 2 - Chemical Exchange. Ann. Rep. NMR Spect. 63, 23-48 (2008).

4. Reeves, L., Shaddick, R., Shaw, K. Nuclear Magnetic Resonance Studies of Multi-site Chemical Exchange. III. Hindered Rotation in Dimethylacetamide, Dimethyl Trifluoro-acetamide, and Dimethyl Benzamide. Can. J. Chem. 49, 3683-3691 (1971).

5. Drakenberg, T., Dahlqvist, K., Forsen, S. Barrier to Internal Rotation in Amides. IV. N,N-Dimethylamides. Substituent and Solvent Effects. J. Phys. Chem. 76, 2178-2183 (1972).

6. Fujiwara, F., Airoldi C. Carbon-13 NMR Study of the Barrier to Internal Rotation of N,N-Dimethylacetamide in the Adduct with Antimony(III) Chloride. J. Phys. Chem. 88, 1640-1642 (1984).

7. Gutowsky, H. S., Holm, C. H. Rate Processes and Nuclear Magnetic Resonance Spectra. II. Hindered Internal Rotation of Amides. J. Chem. Phys. 25, 1228-1234 (1956).

8. Forsen, S., Hoffman, R. A. A New Method for the Study of Moderately Rapid Chemical Exchange Rates Employing Nuclear Magnetic Double Resonance. Acta Chem. Scand. 17, 1787-1788 (1963).

9. Forsen, S., Hoffman, R. A. Study of Moderately Rapid Chemical Exchange Reactions by Means of Nuclear Magnetic Double Resonance. J. Chem. Phys. 39, 2892-2901 (1963).

10. Williams, T. J., Kershaw, A. D., Li, V., Wu, X., An Inversion Recovery NMR Kinetics Experiment. J. Chem. Educ. 88, 665-669 (2011).

11. Bain, A. D., Cramer, J. A., Slow Chemical Exchange in an Eight-Coordinated Bicentered Ruthenium Complex Studied by One-Dimensional Methods. Data Fitting and Error Analysis. J. Magn. Res., Series A. 118, 21-27 (1996).

12. Sandstrom, J., Dynamic NMR Spectroscopy. Academic: New York, ISBN: 01261862009780126186208 (1982).

13. Castanar, L., Nolis, P., Virgili, A., Parella T., Measurement of $T_{1} / T_{2}$ Relaxation Times in Overlapped Regions from Homodecoupled ${ }^{1} \mathrm{H}$ Singlet Signals. J. Magn. Reson. 244, 30-35. (2014).

14. Muñoz, M. P., de la Torre, M. C., Sierra, M. A.New Platinum-Catalysed Dihydroalkoxylation of Allenes. Adv. Synth. Catal. 352, 2189-2194 (2010).

15. Muñoz, M. P., de la Torre, M. C., Sierra, M. A. Platinum-Catalysed Bisindolylation of Allenes: A Complementary Alternative to Gold Catalysis. Chem. Eur. J. 18, 4499-4504 (2012).

16. Hurtado-Rodrigo, C., Hoehne, S., Muñoz, M. P.A New Gold-Catalysed Azidation of Allenes. Chem. Comm. 50, 1494-1496 (2014).

17. Vrieze, K., Volger, H. C., Gronert, M., Praat, A. P. Intramolecular Rearrangements in Platinum- $\pi$-Tetramethylallene Compounds as Influenced by Ligands Trans to the Allene Group. J. Organometal. Chem. 16, P19-P22 (1969).

18. Vrieze, K., Volger, H.C., Praat, A. P. Complexes of Allenes with Platinum (II) and Rhodium (I). J. Organometal. Chem. 21, 467-475 (1970).

19. Brown, T. J., Sugie, A., Leed, M. G. D., Widenhoefer R. A. Structures and Dynamic Solution Behavior of Cationic, Two-Coordinate Gold(I)-mAllene Complexes. Chem. Eur. J. 18, 6959-6971 (2012).

20. Yang, W., Hashmi, S. K. Mechanistic Insights into the Gold Chemistry of Allenes. Chem. Soc. Rev., 43, 2941-2955 (2014).

21. Quiros, M. T., Angulo, J., Munoz, M. P. Kinetics of Intramolecular Chemical Exchange by Initial Growth Rates of Spin Saturation Transfer Difference Experiments (SSTD NMR). Chem. Commun. 51, 10222-10225 (2015).

22. Mayer, M., Meyer, B. Characterization of Ligand Binding by Saturation Transfer Difference NMR Spectroscopy. Ang. Chem. Int. Ed. 38, 1784-1788 (1999).

23. Angulo, J., Nieto, P. STD-NMR: Application to Transient Interactions Between Biomolecules - A Quantitative Approach. Eur. Biophys. J. 40, 1357-1369 (2011).

24. Kemper, S., Patel, M. K., Errey, J. C., Davis, B. G., Jones, J. A., Claridge T. D. W.Group Epitope Mapping Considering Relaxation of the Ligand (GEM-CRL): Including Longitudinal Relaxation Rates in the Analysis of Saturation Transfer Difference (STD) Experiments. J. Magn. Reson. 203, 1-10 (2010).

25. TopSpin program. Bruker Corp., http://www.bruker.com/products/mr/nmr/nmr-software/software/topspin/ (2015).

26. Berger, S., Braun, S. 200 and More NMR Experiments: A Practical Course. Wiley, ISBN: 978-3-527-31067-8 (2004).

27. Cutting, B, Shelke, S. V., Dragic, Z., Wagner, B., Gathje, H., Kelm, S., Ernst, B. Sensitivity Enhancement in Saturation Transfer Difference (STD) Experiments Through Optimized Excitation Schemes. Magn Reson Chem. 45, 720-724 (2007).

28. Ley, N.B., Rowe, M.L., Williamson, R. A., Howard, M. J. Optimising Selective Excitation Pulses To Maximize Saturation Transfer Difference NMR Spectroscopy. RSC Adv. 4, 7347-7351 (2014).

29. Antanasijevic, A., Ramirez, B., Caffrey, M. Comparison of the Sensitivities of WaterLOGSY and Saturation Transfer Difference NMR Experiments. J. Biomol. NMR. 60(1), 37-44 (2014).

30. Origin 6.0 software. OriginLab Corp., http://originlab.com (2016).

31. Jarek, R. L., Flesher, R. J., Shin S. K. Kinetics of Internal Rotation of N,N-Dimethylacetamide: A Spin-Saturation Transfer Experiment. J. Chem. Ed. 74, 978-982 (1997).

32. Forsen, S., Hoffman, R. A. Exchange Rates by Nuclear Magnetic Multiple Resonance. III. Exchange Reactions in Systems with Several Nonequivalent Sites. J. Chem. Phys. 40, 1189-1196 (1964). 